Pacific Journal of Mathematics

TWISTED SELF-HOMOTOPY EQUIVALENCES 


\section{TWISTED SELF-HOMOTOPY EQUIVALENCES}

\section{Allan J. Sieradski}

This paper studies the group $G(A \times B)$ of (homotopy classes of) self-homotopy equivalences of a product $A \times B$ of two connected $C W$ homotopy associative $H$-spaces $A$ and $B$. It establishes the existence of an exact sequence of multiplicative groups

$$
1 \rightarrow[A \wedge B, A \times B] \rightarrow G(A \times B) \rightarrow G L\left(2, \Lambda_{I J}\right) \rightarrow 1
$$

provided that $i \circ[A \times B, A \times B] \circ q \circ[A \wedge B, A \times B]=0$, where $q: A \times B \rightarrow A \wedge B$ is the cofibration induced by the inclusion $i: A \vee B \rightarrow A \times B$ of the sum into the product. The entry $G L\left(2, \Lambda_{I J}\right)$ is the group of invertible matrices

$$
\left(h_{I J}\right)=\left(\begin{array}{ll}
h_{A A} & h_{A B} \\
h_{B A} & h_{B B}
\end{array}\right)
$$

with entries $h_{I J}$ in the homotopy sets $A_{I J}=[I, J]$ for $I, J=$ $A, B$, where matrix multiplication is defined by

$$
\left(h_{I J}\right)\left(k_{I J}\right)=\left(h_{I A} \circ k_{A I}+h_{I B} \circ k_{B J}\right)
$$

in terms of composition $\circ$ and the operation + in the homotopy sets $[I, J]$, and where the multiplicative unit is

$$
\left(\delta_{I J}\right)=\left(\begin{array}{cc}
1_{A} & 0 \\
0 & 1_{B}
\end{array}\right) \text {. }
$$

The homomorphism $G(A \times B) \rightarrow G L\left(2, \Lambda_{I J}\right)$ is given by the correspondence of $h: A \times B \rightarrow A \times B$ with the matrix

$$
\left(\begin{array}{ll}
i_{A} \circ h \circ p_{A} & i_{A} \circ h \circ p_{B} \\
i_{B} \circ h \circ p_{A} & i_{B} \circ h \circ p_{B}
\end{array}\right)
$$

with entries obtained from $h$ by composing with the inclusions

$$
i_{A}: A \rightarrow A \times B \text { and } i_{B}: B \rightarrow A \times B
$$

and the projections

$$
p_{A}: A \times B \rightarrow A \text { and } p_{B}: A \times B \rightarrow B ;
$$

for a preliminary result states that under the hypothesis above $h: A \times B \rightarrow A \times B$ is a homotopy equivalence if and only if the matrix $\left(i_{I} \circ h \circ p_{J}\right)$ is invertible.

A homotopy equivalence $f \times g: A \times B \rightarrow A \times B$ is referred to as untwisted. These determine a subgroup $G(A) \times G(B) \subset$ $G(A \times B)$ which is isomorphic under the homomorphism $G(A \times$ $B) \rightarrow G L\left(2, \Lambda_{I J}\right)$ to the subgroup of diagonal matrices, and so the nondiagonal matrices give measure of the twisted selfhomotopy equivalences $A \times B \rightarrow A \times B$. The extreme case in which all self-homotopy equivalences are untwisted is considered, and it is shown that $G(A) \times G(B)=G(A \times B)$ if and 
only if the homotopy sets $[A, B],[B, A]$, and $[A \wedge B, A \times B]$ are trivial.

Next, four settings are considered in which

$$
i \circ[A \times B, A \times B] \circ q \circ[A \wedge B, A \times B]=0
$$

and the exact sequence is valid. In the last section the dual situation of the group $G(M \vee N)$ of self-homotopy equivalences of a sum $M \vee N$ of two co- $H$-spaces $M$ and $N$ is briefly sketched.

2. The short exact sequence. We work with spaces with base points and we fail to distinguish in our notation between a base-pointpreserving map and its homotopy class. Each set $[X, Y]$, with $Y$ a homotopy inversive, homotopy associative $H$-space, of homotopy classes of base-point-preserving maps receives a group structure whose operation will be denoted additively and will be referred to as "addition." Later various other operations will be considered and referred to as "multiplication."

For the composition of $f: X \rightarrow Y$ and $g: Y \rightarrow Z$ we write

$$
f \circ g: X \rightarrow Z,
$$

displaying the maps (classes) in the order of their application. Since we work with subscripts and matrix-like multiplication, this "nonstandard" notation for composition seems preferable; in any case, since we do not work with elements, expressions of the form $g(f(x))$ which suggest the other order of writing composition do not occur.

Throughout this paper, spaces $A$ and $B$ are connected $C W$ complexes which admit homotopy inversive, homotopy associative multiplications $m_{A}: A \times A \rightarrow A$ and $m_{B}: B \times B \rightarrow B$. So it is easy to define some twisted classes $A \times B \rightarrow A \times B$; given $h_{I J}: I \rightarrow J$ for $I, J=A, B$ we define $\left\{\left(h_{I J}\right)\right\}: A \times B \rightarrow A \times B$ by setting the projection onto the $J^{\text {th }}$ factor equal to

$$
h_{A J} \times h_{B J} \circ m_{J}=p_{A} \circ h_{A J}+p_{B} \circ h_{B J}: A \times B \rightarrow J \times J \rightarrow J
$$

for $J=A, B$. For example, if $\delta_{I J}: I \rightarrow J$ is $1: I \rightarrow J$ when $I=J$ and $0: I \rightarrow J$ when $I \neq J$, then $\left\{\left(\delta_{I J}\right)\right\}=1: A \times B \rightarrow A \times B$.

We now consider the extent to which an arbitrary homotopy class $A \times B \rightarrow A \times B$ differs from one of this special form $\left\{\left(h_{I J}\right)\right\}$. Since the product $A \times B$ inherits from $A$ and $B$ a "coordinate-wise" multiplication which is homotopy inversive and homotopy associative, we obtain from the mapping cone sequence for the inclusion $i: A \vee B \rightarrow$ $A \times B$ a short exact sequence of additive groups of homotopy classes

$$
0 \longrightarrow[A \wedge B, A \times B] \stackrel{q^{\sharp}}{\longrightarrow}[A \times B, A \times B] \stackrel{i^{\sharp}}{\longrightarrow}[A \vee B, A \times B] \longrightarrow 0,
$$


where $q: A \times B \rightarrow A \wedge B$ is the inclusion of $A \times B$ onto the base of the mapping cone of $i$. It proves convenient to identify [ $A \vee B, A \times B$ ] with the set of $2 \times 2$ matrices

$$
\left(h_{I J}\right)=\left(\begin{array}{ll}
h_{A A} & h_{A B} \\
h_{B A} & h_{B B}
\end{array}\right)
$$

with entries $h_{I J}$ from the homotopy sets $[I, J]$ for $I, J=A, B$, via the correspondence of $h: A \vee B \rightarrow A \times B$ with the matrix $\left(i_{I} \circ h \circ p_{J}\right)$, where $i_{I}: I \rightarrow A \vee B(I=A, B)$ are the two inclusions of the summands into the sum and $p_{J}: A \times B \rightarrow J(J=A, B)$ are the two projections of the product onto the factors.

Note that given the matrix $\left(h_{I J}\right)$, the class $\left\{\left(h_{I J}\right)\right\}: A \times B \rightarrow A \times B$ defined earlier satisfies

$$
i \circ\left\{\left(h_{I J}\right)\right\}=\left(h_{I J}\right): A \vee B \rightarrow A \times B
$$

because

$$
\begin{aligned}
i_{I} \circ i \circ\left\{\left(h_{I J}\right)\right\} \circ p_{J} & =i_{J} \circ i \circ h_{A J} \times h_{B J} \circ m_{J} \\
& =i_{I} \circ h_{A J} \vee h_{B J} \circ i \circ m_{J} \\
& =i_{I} \circ h_{A J} \vee h_{B J} \circ \nabla \\
& =h_{I J} .
\end{aligned}
$$

Therefore, for any $h: A \times B \rightarrow A \times B$ we have $h-\left\{\left(h_{I J}\right)\right\}$ in kernel $i^{\sharp}=$ image $q^{\sharp}$, if we choose $h_{I J}=i_{I} \circ i \circ h \circ p_{J}$ for $I, J=A, B$. This proves

Lemma 1. Each class $h: A \times B \rightarrow A \times B$ is of the form

$$
h=q \circ f+\left\{\left(h_{I J}\right)\right\} \text {. }
$$

To return to the investigation of $G(A \times B)$ we observe that composition of homotopy classes determines an associative operation in $[A \times B, A \times B]$ which we will write multiplicatively. This operation has a unit 1 , is generally noncommutative, and distributes over addition from one side: $f \circ(g+h)=f \circ g+f \circ h$. Thus $[A \times B, A \times B]$ is nearly a ring (lacking commutativity of addition and left distributivity of multiplication), and the group $G(A \times B)$ of self-homotopy equivalences of $A \times B$ is just the group of invertible elements of $[A \times B$, $A \times B]$ with respect to the multiplication.

To abstract information about $G(A \times B)$ from the additive exact sequence above, we try to introduce compatible multiplications in its other entries. The identification of the set $[A \vee B, A \times B]$ with the set of $2 \times 2$ matrices $\left(h_{I J}\right)$ with entries $h_{I J}$ from the homotopy sets $[I, J]$ for $I, J=A, B$ makes it possible to introduce matrix multiplication in $[A \vee B, A \times B]$ : 


$$
\left(h_{I J}\right) \cdot\left(k_{I J}\right)=\left(h_{I A} \circ k_{A J}+h_{I B} \circ k_{B J}\right)
$$

where the indicated addition takes place in $[I, J]$. This matrix multiplication need not be associative, but does admit a unit $\left(\delta_{I J}\right)$ and so we can refer to invertible matrices $\left(h_{I J}\right): A \vee B \rightarrow A \times B$. The next theorem begins to relate these invertible matrices $\left(h_{I J}\right): A \vee B \rightarrow A \times B$ to homotopy equivalences $h: A \times B \rightarrow A \times B$.

THEOREM 2. If $i \circ h=\left(h_{I J}\right): A \vee B \rightarrow A \times B$ is an invertible matrix, then $h: A \times B \rightarrow A \times B$ is a homotopy equivalence. In particular, each invertible matrix $\left(h_{I J}\right)$ determines a homotopy equivalence $\left\{\left(h_{I J}\right)\right\} ; A \times B \rightarrow A \times B$.

We base the proof on the following facts.

Lemma 3. (i) If we identify $[X, A \times B]$ with the set of $1 \times 2$ matrices $\left(g_{J}\right)$ with entries $g_{J}: X \rightarrow J(J=A, B)$ by means of the correspondence $g \rightarrow\left(g \circ p_{J}\right)$, then $\left\{\left(h_{I J}\right)\right\}_{\sharp}:[X, A \times B] \rightarrow[X, A \times B]$ can be calculated by matrix multiplication

$$
\left(g_{J}\right) \circ\left\{\left(h_{I J}\right)\right\}=\left(g_{A} \circ h_{A J}+g_{B} \circ h_{B J}\right)
$$

(ii) If $X$ has a comultiplication and $i \circ h=\left(h_{I J}\right)$, then $h_{\sharp}=\left\{\left(h_{\overline{I J}}\right)\right\}_{\sharp}$ : $[X, A \times B] \rightarrow[X, A \times B]$.

(iii) If $X$ has a comultiplication, then $\left\{\left(h_{I J}\right)\right\}_{\sharp} \circ\left\{\left(k_{I J}\right)\right\}_{\sharp}=\left\{\left(h_{I J}\right) \cdot\left(k_{I J}\right)\right\}_{\sharp}$ : $[X, A \times B] \rightarrow[X, A \times B]$.

Proof. Since $\left\{\left(h_{I J}\right)\right\} \circ p_{J}=p_{A} \circ h_{A J}+p_{B} \circ h_{B J}$, we have for $g: X \rightarrow$ $A \times B$

$$
\begin{aligned}
g \circ\left\{\left(h_{I J}\right)\right\} \circ p_{J} & =g \circ\left(p_{A} \circ h_{A J}+p_{B} \circ h_{B J}\right) \\
& =g \circ p_{A} \circ h_{A J}+g \circ p_{B} \circ h_{B J}
\end{aligned}
$$

which proves (i). Now if $X$ has a comultiplication then $i_{\sharp}:[X, A \vee B] \rightarrow$ $[X, A \times B]$ is surjective so $g=d \circ i$. If we use Lemma 1 , then we see that

$$
\begin{aligned}
g \circ h & =g \circ\left(q \circ f+\left\{\left(h_{I J}\right)\right\}\right)=g \circ q \circ f+g \circ\left\{\left(h_{I J}\right)\right\} \\
& =d \circ i \circ q \circ f+g \circ\left\{\left(h_{I J}\right)\right\}=g \circ\left\{\left(h_{I J}\right)\right\}
\end{aligned}
$$

which proves (ii). If $X$ has a comultiplication then the usual addition in $[X, J](J=A, B)$ is abelian and furthermore coincides with the operation induced by the comultiplication so that each $k_{I J \sharp}:[X, I] \rightarrow$ $[X, J]$ is a homomorphism. Thus we have

$$
\left(g_{J}\right) \circ\left\{\left(h_{I J}\right)\right\} \circ\left\{\left(k_{I J}\right)\right\}=\left(g_{A} \circ h_{A J}+g_{B} \circ h_{B J}\right) \circ\left\{\left(k_{I J}\right)\right\}
$$




$$
\begin{aligned}
& =\left(\left(g_{A} \circ h_{A A}+g_{B} \circ h_{B A}\right) \circ k_{A J}+\left(g_{A} \circ h_{A B}+g_{B} \circ h_{B B}\right) \circ k_{B J}\right) \\
& =\left(g_{A} \circ h_{A A} \circ k_{A J}+g_{B} \circ h_{B A} \circ k_{A J}+g_{A} \circ h_{A B} \circ k_{B J}+g_{B} \circ h_{B B} \circ k_{B J}\right) \\
& =\left(g_{A} \circ h_{A A} \circ k_{A J}+g_{A} \circ h_{A B} \circ k_{B J}+g_{B} \circ h_{B A} \circ k_{A J}+g_{B} \circ h_{B B} \circ k_{B J}\right) \\
& =\left(g_{A} \circ\left(h_{A A} \circ k_{A J}+h_{A B} \circ k_{B J}\right)+g_{B} \circ\left(h_{B A} \circ k_{A J}+h_{B B} \circ k_{B J}\right)\right) \\
& =\left(g_{J}\right) \circ\left\{\left(h_{I J}\right) \cdot\left(k_{I J}\right)\right\}
\end{aligned}
$$

which proves (iii).

Proof of Theorem 2. It is an immediate consequence of (i) and (iii) of the lemma that for an invertible matrix $\left(h_{I J}\right)$ the function

$$
\left\{\left(h_{I J}\right)\right\}_{\sharp}[X, A \times B] \rightarrow[X, A \times B]
$$

is an isomorphism, provided that $X$ is comultiplicative. In view of (ii) and the fact that the spheres $S^{k}(k \geqq 1)$ are comultiplicative, the hypothesis that $i \circ h=\left(h_{I J}\right)$ is an invertible matrix then implies that

$$
h_{\sharp}: \pi_{k}(A \times B) \rightarrow \pi_{k}(A \times B)
$$

is an isomorphism ( $k \geqq 1)$. So the Whitehead Theorem and our hypothesis that $A$ and $B$ are connected $C W$ complexes allow us to conclude that $h: A \times B \rightarrow A \times B$ is a homotopy equivalence.

A crucial consideration, which we postpone until the next section, is whether

$$
i^{\sharp}:[A \times B, A \times B] \rightarrow[A \vee B, A \times B]
$$

is a homomorphism from the composition multiplication to the matrix multiplication. When $i^{\#}$ is a multiplicative homomorphism, its surjectivity shows that matrix multiplication is associative and hence the set $G L\left(2, \Lambda_{I J}\right)$ of invertible matrices $\left(h_{I J}\right)$ with entries $h_{I J} \in \Lambda_{I J}=[I, J]$ $(I, J=A, B)$ is a group under matrix multiplication.

Theorem 4. Let $i^{\sharp}:[A \times B, A \times B] \rightarrow[A \vee B, A \times B]$ be a multiplicative homomorphism. Then

(i) $h: A \times B \rightarrow A \times B$ is a homotopy equivalence if and only if $i \circ h=\left(h_{I J}\right): A \vee B \rightarrow A \times B$ is an invertible matrix, and

(ii) there is a short exact sequence of multiplicative groups

$$
1 \longrightarrow[A \wedge B, A \times B] \stackrel{q^{\sharp}+1}{\longrightarrow} G(A \times B) \stackrel{i^{\#}}{\longrightarrow} G L\left(1, \Lambda_{I J}\right) \longrightarrow 1 .
$$

Proof. (i) Theorem 2 states that if $i \circ h$ is an invertible matrix, then $h$ is a homotopy equivalence. The converse holds here since invertible elements are sent into invertible elements by the multipli- 
cative homomorphism $i^{\sharp}$.

(ii) It follows from (i) that the restriction of the multiplicative homomorphism $i^{\sharp}$ to $G(A \times B) \subset[A \times B, A \times B]$ is a surjective multiplicative group homomorphism

$$
i^{\sharp}: G(A \times B) \rightarrow G L\left(2, \Lambda_{I J}\right) .
$$

The kernel of this homomorphism consists of those $h \in G(A \times B)$ with $h-1 \in$ kernel $i^{\sharp}=$ image $q^{\sharp}$. Thus the multiplicative kernel is image $q^{\sharp}+1$ with the multiplicative structure described by

$$
(q \circ f+1) \cdot(q \circ g+1)=(q \circ f+1) \circ q \circ g+q \circ f+1 .
$$

We can therefore consider the multiplicative kernel as the image of the injection

$$
q^{\sharp}+1:[A \wedge B, A \times B] \rightarrow G(A \times B)
$$

and this is a multiplicative group homomorphism if the domain is given the multiplication uniquely described by the requirement

$$
q \circ(f \circ g)=(q \circ f+1) \circ q \circ g+q \circ f .
$$

There is a situation in which the addition in $[A \wedge B, A \times B]$ coincides with the multiplication just introduced. Suppose that $i$ : $A \vee B \rightarrow A \times B$ is the cofibration induced by some map $\alpha: X \rightarrow A \vee B$ that is, $A \times B=(A \vee B) \bigcup_{\alpha} C X=C_{\alpha}$, the mapping cone of $\alpha$, and the sequence

$$
A \vee B \stackrel{i}{\longrightarrow} A \times B \stackrel{q}{\longrightarrow} A \wedge B
$$

is equivalent to the tail-end of the sequence

$$
X \stackrel{\alpha}{\longrightarrow} A \vee B \stackrel{i(\alpha)}{\longrightarrow} C_{\alpha} \stackrel{j(\alpha)}{\longrightarrow} \sum X
$$

where $i(\alpha): A \vee B \rightarrow C_{\alpha}$ and $j(\alpha): C_{\alpha} \rightarrow C_{\alpha} /(A \vee B)=\sum X$ are the indicated inclusion and quotient maps. Then there is a cooperation

$$
c: A \times B \rightarrow(A \wedge B) \vee(A \times B)
$$

in the sense that

$$
\begin{aligned}
& 1 \simeq c \circ p_{2}: A \times B \rightarrow(A \wedge B) \vee(A \times B) \rightarrow A \times B \\
& q \simeq c_{\circ} p_{1}: A \times B \rightarrow(A \wedge B) \vee(A \times B) \rightarrow A \wedge B .
\end{aligned}
$$

The cooperation is essentially the map $C_{\alpha} \rightarrow \sum X \vee C_{\alpha}$ which collapses the equatorial belt $X \times 1 / 2$ in the cone $C X$.

Proposition 5. When $i: A \vee B \rightarrow A \times B$ is an induced cofibration 
then

$$
q^{\sharp}+1:[A \wedge B, A \times B] \rightarrow[A \times B, A \times B]
$$

is a homomorphism from the additive operation in $[A \wedge B, A \times B]$ to the composition multiplication in $[A \times B, A \times B]$.

Proof. For $f \in[A \wedge B, A \times B]$ and $h \in[A \times B, A \times B]$, define

$$
h^{f}=c \circ f \vee h \circ \nabla: A \times B \rightarrow A \times B .
$$

Since $A \times B$ has a multiplication $n$,

$$
\begin{aligned}
h^{f} & =c \circ f \vee h \circ \nabla \\
& =c \circ f \vee h \circ j \circ n, \quad \text { for } j:(A \times B) \vee(A \times B) \rightarrow(A \times B) \times(A \times B), \\
& =c \circ j^{\prime} \circ f \times h \circ n, \quad \text { for } j^{\prime}:(A \wedge B) \vee(A \times B) \rightarrow(A \wedge B) \times(A \times B), \\
& =\Delta \circ q f \times h \circ n, \quad \text { since } q \simeq c \circ p_{1} \text { and } 1 \simeq c \circ p_{2}, \\
& =q f+h .
\end{aligned}
$$

So for $f, g \in[A \wedge B, A \times B]$, and $h, k \in[A \times B, A \times B]$ we have

$$
\begin{aligned}
h^{f} \cdot k^{g} & =(c \circ f \vee h \circ \nabla) \circ k^{g} \\
& =c \circ\left(f \circ k^{g} \vee h \circ k^{g}\right) \circ \nabla \\
& =q \circ f \circ k^{g}+h \circ k^{g} \\
& =q \circ f \circ(q \circ g+k)+h \circ(q \circ g+k) \\
& =q \circ f \circ q \circ g+q \circ f \circ k+h \circ q \circ g+h \circ k \\
& =q \circ f \circ k+h \circ q \circ g+h \circ k,
\end{aligned}
$$

as the presence of a comultiplication on $A \wedge B=\sum X$ guarantees that $f=e \circ i: A \wedge B \rightarrow A \vee B \rightarrow A \times B$ for a suitable $e: A \wedge B \rightarrow A \vee B$, and so $f \circ q=e \circ i \circ q=e \circ 0=0$. In particular

$$
\begin{aligned}
(q \circ f+1) \cdot(q \circ g+1) & =1^{f} \cdot 1^{g} \\
& =q \circ f+q \circ g+1 \\
& =q \circ(f+g)+1
\end{aligned}
$$

which proves the proposition.

While this result does not have great applicability, it is tailored to the case $A=S^{n}, B=S^{m}$ for $n, m$ from the collection $(1,3,7)$ for then we have the mapping cone sequence

$$
S^{n+m-1} \stackrel{\alpha}{\longrightarrow} S^{n} \vee S^{m} \stackrel{i}{\longrightarrow} S^{n} \times S^{m} \stackrel{q}{\longrightarrow} S^{n} \wedge S^{m} .
$$

3. The multiplicative homomorphism. We see that $i^{\sharp}\left(\left\{\left(h_{I J}\right)\right\}\right.$ • $\left.\left\{\left(k_{I J}\right)\right\}\right)=i^{\sharp}\left\{\left(h_{I J}\right)\right\} \cdot i^{\sharp}\left\{\left(k_{I J}\right)\right\}$ since 


$$
\begin{aligned}
& i_{I} \circ i \circ\left\{\left(h_{I J}\right)\right\} \circ\left\{\left(k_{I J}\right)\right\} \circ p_{J} \\
&=i_{I} \circ\left(h_{I J}\right) \circ\left(p_{A} \circ k_{A J}+p_{B} \circ k_{B J}\right) \\
&=i_{I} \circ\left(h_{I J}\right) \circ p_{A} \circ k_{A J}+i_{I} \circ\left(h_{I J}\right) \circ p_{B} \circ k_{B J} \\
&=h_{I A} \circ k_{A J}+h_{I B} \circ k_{B J} \\
&=i_{I} \circ\left(h_{I J}\right) \cdot\left(k_{I J}\right) \circ p_{J} .
\end{aligned}
$$

For the general case $h=q \circ f+\left\{\left(h_{I J}\right)\right\}$ and $k=q \circ g+\left\{\left(k_{I J}\right)\right\}$, we have

$$
i^{\sharp}(h \cdot k)=i \circ h \circ q \circ g+i^{\sharp}(h) \cdot i^{\sharp}(k),
$$

since

$$
\begin{aligned}
i \circ h \circ k & =i \circ h \circ\left(q \circ g+\left\{\left(k_{I J}\right)\right\}\right) \\
& =i \circ h \circ q \circ g+i \circ\left\{\left(h_{I J}\right)\right\} \circ\left\{\left(k_{I J}\right)\right\} .
\end{aligned}
$$

We record this fact as follows.

Theorem 6. The function $i^{\ddagger}:[A \times B, A \times B] \rightarrow[A \vee B, A \times B]$ is a multiplicative homomorphism if and only if

$$
i \circ[A \times B, A \times B] \circ q \circ[A \wedge B, A \times B]=0,
$$

or equivalently, kernel $i^{\sharp}=q \circ[A \wedge B, A \times B]$ is a right ideal in. $[A \times B, A \times B]$.

Corollary 7. If the $H$-spaces $A$ and $B$ satisfy $[A \wedge B, A \times B]=$ 0 , then the group of self-homotopy equivalences of $A \times B$ is $G L\left(2, \Lambda_{I J}\right)$, the group of invertible matrices $\left(h_{I J}\right)$ with entries $h_{I J} \in \Lambda_{I J}=[I, J]$, for $I, J=A, B$.

CoRollary 8. If either

(i) the $H$-spaces $A$ and $B$ admit comultiplications,

(ii) all maps $I \rightarrow J(I, J=A, B)$ which induce the zero homomorphism on homotopy are null-homotopic, or

(iii) the homotopy set $[A \vee B, A \wedge B]$ is trivial, then

(vi) a map $h: A \times B \rightarrow A \times B$ is a homotopy equivalence if and: only if $i \circ h=\left(h_{I J}\right): A \vee B \rightarrow A \times B$ is an invertible matrix, and

$(\mathrm{v})$ there is a short exact sequence of multiplicative groups

$$
1 \rightarrow[A \wedge B, A \times B] \rightarrow G(A \times B) \rightarrow G L\left(2, \Lambda_{I J}\right) \rightarrow 1 .
$$

Corollary 9. For any two $H$-spaces $A$ and $B$

$$
G(A) \times G(B)=G(A \times B)
$$

if and only if the homotopy sets $[A, B],[B, A]$, and $[A \wedge B, A \times B]$ are trivial. 
Proof of the corollaries. Corollary 7 and Corollary 8 (iii) are immediate consequences of Theorems 4 and 6. For Corollary 8 (i) and (ii) we point out that if $X$ is comultiplicative

$$
\begin{gathered}
{[X, A \vee B] \circ i_{\circ}[A \times B, A \times B] \circ q \circ[A \wedge B, A \times B]} \\
\subset[X, A \vee B] \circ i \circ q \circ[A \wedge B, A \times B]=0 .
\end{gathered}
$$

Thus, if $A$ and $B$ admit comultiplications, then

$$
i_{I} \circ i \circ[A \times B, A \times B] \circ q \circ[A \wedge B, A \times B]=0
$$

for $I=A, B$, which proves Corollary 8 (i). In any case each map of

$$
i_{1} \circ i \circ[A \times B, A \times B] \circ q \circ[A \wedge B, A \times B] \circ p_{J}
$$

induces the zero function $[X, I] \rightarrow[X, J](I, J=A, B)$ provided $X$ is comultiplicative. If all maps $I \rightarrow J(I, J=A, B)$ which induce the zero homomorphism on homotopy are null-homotopic, then the above maps are null, which proves Corollary 8 (ii).

For Corollary 9 note that if $G(A) \times G(B)=G(A \times B)$ we obtain an exact sequence as in Theorem 4 . From the injectivity of $G(A) \times$ $G(B) \rightarrow G L\left(2, \Lambda_{I J}\right)$ and exactness we conclude $[A \wedge B, A \times B]=0$; from its surjectivity we conclude $[A, B]=0=[B, A]$. Conversely, if $[A, B]$, $[B, A]$, and $[A \wedge B, A \times B]$ are trivial we have from Corollary 7 and direct calculation $G(A \times B)=G L\left(2, \Lambda_{I J}\right)=G(A) \times G(B)$.

EXAMPLES. 1. For integers $n, m \geqq 1$ and abelian groups $G$ and $H$, the Eilenberg-MaLane spaces $A=K(G, n)$ and $B=K(H, m)$ are $H$-spaces and $[A \wedge B, A \times B]=0$ so that by Corollary $7, G(K(G, n) \times$ $K(H, m))=G L\left(2, \Lambda_{I J}\right)$. If $n=m$, then $\Lambda_{I J}=\operatorname{Hom}_{Z}(I, J)(I, J=G, H)$ : if in addition $G=H$, then $G(K(G, n) \times K(G, n)=G L(2, \Lambda)$, the general linear group of degree 2 over the endomorphism ring $A=\operatorname{Hom}_{Z}(G, G)$ of the abelian group $G$. If $n>m$, then $\Lambda_{A B}=H^{m}(G, n ; H)=0$ and so $G L\left(2, \Lambda_{I J}\right)$ consists of triangular matrices $\left(h_{I J}\right)$ with entries $h_{A A} \in$ Iso $(G, G), h_{B A} \in H^{n}(H, m ; G)$, and $h_{B B} \in$ Iso $(H, H)$. We write the group $G L\left(2, \Lambda_{I J}\right)$ of such triples with matrix multiplication as

$$
G(K(G, n) \times K(H, m))=\operatorname{Iso}(G, G) * H^{n}(H, m ; G) * \operatorname{Iso}(H, H) .
$$

2. If $X$ is an $n$-connected $C W$ complex with $\pi_{k}(X)=0$ for $k>$ $2 n$, then the group $G(X)$ of self-homotopy equivalences of $X$ is the group of units of the ring $A=[X, X]$, while the group $G(X \times X)$ of self-homotopy equivalences of the product $X \times X$ is the general linear group $G L(2, \Lambda)$ of degree 2 over $\Lambda$. So the situation here is like that which occurs in the entirely algebraic setting. That $A=[X, X]$ is a ring follows from the facts that (i) as $i^{\sharp}:[X \times X, X] \rightarrow[X \vee X, X]$ 
is a bijection, $X$ is a homotopy commutative $H$-space for which each self-map $X \rightarrow X$ is an $H$-map, (ii) as $i^{\sharp}:[X \times X \times X, X] \rightarrow[X \vee X \vee$ $X, X]$ is a bijection, the multiplication is homotopy associative, and (iii) as $X$ is a $C W$ complex, the multiplication is homotopy inversive. Alternatively, we could argue from the fact that $X$ has the homotopy type of an infinite loop space and each self map can be achieved up to homotopy as a looped map. Finally, that $G(X \times X)=G L(2, \Lambda)$ follows from Corollary 7 as $[X \wedge X, X \times X]=0$.

3. If $A=S^{n}=B(n=1,3,7)$, then the additive and multiplicative structure of $\Lambda_{I J}=\left[S^{n}, S^{n}\right](I, J=A, B)$ used in defining matrix multiplication coincides that that of the ring $Z$ of integers under the usual isomorphism $\left[S^{n}, S^{n}\right] \approx Z$. Thus $G L\left(2, \Lambda_{I J}\right)=G L(2, Z)$, the group of $2 \times 2$ matrices with integer entries and determinant \pm 1 . We have from Corollary 8 that

$$
h: S^{n} \times S^{n} \rightarrow S^{n} \times S^{n} \quad(n=1,3,7)
$$

is a homotopy equivalence if and only if the matrix $i \circ h=\left(h_{I J}\right): S^{n} \vee$ $S^{n} \rightarrow S^{n} \times S^{n}$ of "integers" has determinant \pm 1 .

Since the additive structure in $\left[S^{n} \wedge S^{n}, S^{n} \times S^{n}\right]$ coincides with that of

$$
\pi_{2 n}\left(S^{n} \times S^{n}\right)= \begin{cases}0 & n=1 \\ Z_{12} \oplus Z_{12} & n=3 \\ Z_{120} \oplus Z_{120} & n=7\end{cases}
$$

Corollary 7 shows that $G\left(S^{1} \times S^{1}\right)=G L(2, Z)$, while Proposition 5 and Corollary 8 yield exact sequences

$$
0 \rightarrow Z_{12} \oplus Z_{12} \rightarrow G\left(S^{3} \times S^{3}\right) \rightarrow G L(2, Z) \rightarrow 1
$$

and

$$
0 \rightarrow Z_{120} \oplus Z_{120} \rightarrow G\left(S^{7} \times S^{7}\right) \rightarrow G L(2, Z) \rightarrow 1 .
$$

4. If $A=S^{1}, B=S^{n}(n=3,7)$, then $\Lambda_{I J}=Z$ for $I=J$ and $=0$ for $I \neq J$. This implies that $G L\left(2, \Lambda_{I J}\right)$ consists of the four invertible diagonal matrices with integer entries, which makes it isomorphic to the (abelian) subgroup $G\left(S^{1}\right) \times G\left(S^{n}\right) \subset G\left(S^{1} \times S^{n}\right)$, and so the exact sequence of Corollary 8 is split. In view of Proposition 5 and the fact that the additive structure of $\left[S^{1} \wedge S_{n}, S^{1} \times S^{n}\right]$ coincides with that of

$$
\pi_{n+1}\left(S^{1} \times S^{n}\right)= \begin{cases}\pi_{4}\left(S^{3}\right)=Z_{2}, & n=3, \\ \pi_{8}\left(S^{7}\right)=Z_{2}, & n=7,\end{cases}
$$


this sequence takes the form

$$
0 \rightarrow Z_{2} \rightarrow G\left(S^{1} \times S^{n}\right) \rightarrow Z_{2} \oplus Z_{2} \rightarrow 0,
$$

where we have used the additive notation $G L\left(2, \Lambda_{I J}\right)=Z_{2} \oplus Z_{2}$.

To prove that $G\left(S^{1} \times S^{n}\right)$ is abelian we first note that for

$$
f, g \in\left[S^{1} \wedge S^{n}, S^{1} \times S^{n}\right]=Z_{2},\left\{\left(h_{I J}\right)\right\},\left\{\left(k_{I J}\right)\right\} \in G\left(S^{1} \times S^{n}\right)
$$

we have $f \circ\left\{\left(k_{I J}\right)\right\}=0$ if and only if $f=0$ and therefore $q \circ f \circ\left\{\left(k_{I J}\right)\right\}=$ $q \circ f$, while $\left\{\left(h_{I J}\right)\right\} \circ q \circ g=h_{A A} \times h_{B B} \circ q \circ g=q \circ h_{A A} \wedge h_{B B} \circ g=0$ if and only if $g=0$ and therefore $\left\{\left(h_{I J}\right)\right\} \circ q \circ g=q \circ g$. Then the operation

$$
\begin{aligned}
(q \circ f+ & \left.\left\{\left(h_{I J}\right)\right\}\right) \cdot\left(q \circ g+\left\{\left(k_{I J}\right)\right\}\right)=\left\{\left(h_{I J}\right)\right\}^{f} \cdot\left\{\left(k_{I J}\right)\right\}^{g} \\
& =q \circ f \circ\left\{\left(k_{I J}\right)\right\}+\left\{\left(h_{I J}\right)\right\} \circ q \circ g+\left\{\left(h_{I J}\right)\right\}\left\{\left(k_{I J}\right)\right\}, \\
& \quad \text { as in Proposition 5, } \\
& =q \circ f+q \circ g+\left\{\left(h_{I J}\right)\right\}\left\{\left(k_{I J}\right)\right\} \\
& =q \circ(f+g)+\left\{\left(h_{I J}\right)\right\}\left\{\left(k_{I J}\right)\right\}
\end{aligned}
$$

in $G\left(S^{1} \times S^{n}\right)$ is observed to be abelian and we conclude

$$
G\left(S^{1} \times S^{n}\right) \approx Z_{2} \oplus Z_{2} \oplus Z_{2} \quad(n=3,7) .
$$

5. If $A=S^{3}$ and $B=S^{7}$ we obtain the exact sequence

$$
0 \rightarrow Z_{15} \oplus Z_{24} \rightarrow G\left(S^{3} \times S^{7}\right) \rightarrow Z_{2} \oplus Z_{2} \oplus Z_{2} \rightarrow 0
$$

in view of the data $\pi_{10}\left(S^{3}\right)=Z_{15}, \pi_{10}\left(S^{7}\right)=Z_{24}$, and $\pi_{7}\left(S^{3}\right)=Z_{2}$, and the fact that $G L\left(2, \Lambda_{I J}\right)=( \pm 1) * \pi_{7}\left(S^{3}\right) *( \pm 1)$ (see Example 1 for notation) can be written additively as $Z_{2} \oplus Z_{2} \oplus Z_{2}$. Associated with the nonzero element $h_{B A} \in \pi_{7}\left(S^{3}\right)=Z_{2}$ there are four basic twisted selfhomotopy equivalences $\left\{\left(h_{I J}\right)\right\}: S^{3} \times S^{7} \rightarrow S^{3} \times S^{7}$ given by

$$
h_{A A}= \pm 1 \in\left[S^{3}, S^{3}\right], h_{B B}= \pm 1 \in\left[S^{7}, S^{7}\right]
$$

and each of these has $15 \cdot 24$ variations of the form $q \circ g+\left\{\left(h_{I J}\right)\right\}$ for $g \in\left[S^{3} \wedge S^{7}, S^{3} \times S^{7}\right]=\pi_{10}\left(S^{3}\right) \oplus \pi_{10}\left(S^{7}\right)=Z_{15} \oplus Z_{24}$.

6. We consider here the case $A=P^{3}=B$ (real projective 3 -space). The space $P^{3}$ cannot admit a comultiplication since spaces with both a multiplication and a comultiplication have fundamental group $\pi_{1}=0$ or $Z$. Thus this situation doesn't fit into Corollary 8 (i), but it is covered by 8 (ii). It is known that two maps $P^{3} \rightarrow P^{3}$ are homotopic if and only if they induce the same homomorphism $\phi$ on $\pi_{1}=Z_{2}=$ $\left(\omega^{0}, \omega^{1}\right)$ and have the same degree. Since the degree of $f: P^{3} \rightarrow P^{3}$ can be calculated from $f_{\sharp}: \pi_{3}\left(P^{3}\right) \rightarrow \pi_{3}\left(P^{3}\right)$, it follows that hypothesis 8 (ii) is satisfied. 
Moreover, there exists a map $P^{3} \rightarrow P^{3}$ of degree $d$ inducing $\phi$ : $Z_{2} \rightarrow Z_{2}, \phi\left(\omega^{1}\right)=\omega^{k}(k=0,1)$ if and only if $d \equiv k \bmod 2$. Thus the assignment $f \rightarrow \operatorname{deg} f$ determines a bijection $\left[P^{3}, P^{3}\right] \rightarrow Z$, which is a homomorphism from addition and composition multiplication to integer addition and multiplication. Thus Corollary 8 (ii) provides an exact sequence

$$
1 \rightarrow\left[P^{3} \wedge P^{3}, P^{3} \times P^{3}\right] \rightarrow G\left(P^{3} \times P^{3}\right) \rightarrow G L(2, Z) \rightarrow 1 .
$$

7. If $A=S^{7}$ and $B=S^{3} \times S^{3}$, then $\Lambda_{B A}=0$ and so $G L\left(2, \Lambda_{I J}\right)$ consists of triangular matrices and can be denoted by

$$
G\left(S^{3} \times S^{3}\right) * \pi_{7}\left(S^{3} \times S^{3}\right) *( \pm 1) \text {. }
$$

Since $[A \vee B, A \wedge B]=0$, Corollary 8 (iii) is applicable and provides $1 \rightarrow\left[S^{7} \wedge\left(S^{3} \times S^{3}\right), S^{7} \times S^{3} \times S^{3}\right] \rightarrow G\left(S^{7} \times S^{3} \times S^{3}\right) \rightarrow G L\left(2, \Lambda_{I J}\right) \rightarrow 1$.

REMARK. In Examples 3, 4, 5, and 7 there occurs the $H$-space $S^{7}$ none of whose multiplications is homotopy associative! But at least the standard one induced by the multiplication of Cayley numbers is diassociative in that the substructure generated by any two elements of $\left[X, S^{7}\right]$ is associative. Fortunately, this is sufficient to provide the implication

$$
h-\left\{\left(h_{I J}\right)\right\}=q \circ f \Rightarrow h=q \circ f+\left\{\left(h_{I J}\right)\right\}
$$

needed for Lemma 1 , the implication

$$
h-1 \in \text { image } q^{\sharp} \Longrightarrow h \in \text { image } q^{\ddagger}+1
$$

needed for Theorem 4 (ii), and, together with the cyclicity of $\left[S^{n} \wedge\right.$ $\left.S^{7}, S^{7}\right]$ for $n=1,3,7$, the equality

$$
q \circ f+(q \circ g+1)=(q \circ f+q \circ g)+1
$$

needed for Proposition 5-the only places where associativity is crucial and does not follow from other considerations.

4. The dual case. Let $M$ and $N$ be simply connected $C W$ complexes which admit homotopy inversive, homotopy associative comultiplications $c_{M}: M \rightarrow M \vee M$ and $c_{N}: N \rightarrow N \vee N$. Again the operation of the induced group structures on the homotopy sets will be written additively.

Given four maps $h_{I J}: I \rightarrow J(I, J=M, N)$ we can define a map $\left\langle\left(h_{I J}\right)\right\rangle: M \vee N \rightarrow M \vee N$ by $i_{I} \circ\left\langle\left(h_{I J}\right)\right\rangle=c_{I} \circ h_{I M} \vee h_{I N}$. To measure the deviation of an arbitrary map $h: M \vee N \rightarrow M \vee N$ from this special form we introduce the exact sequence of additive groups 
$0 \longrightarrow[M \vee N, M b N] \stackrel{p_{\sharp}}{\longrightarrow}[M \vee N, M \vee N] \stackrel{i_{\sharp}}{\longrightarrow}[M \vee N, M \times N] \longrightarrow 0$

where the group structures are inherited from the coordinate-wise comultiplication on $M \vee N$ and the map $p: M b N \rightarrow M \vee N$ is the fibration induced by $i: M \vee N \rightarrow M \times N$. Since $i_{\sharp}\left\langle\left(h_{I J}\right)\right\rangle=\left(h_{I J}\right)$, we can use the exactness of the above sequence to show

Lemma 1'. Each class $h: M \vee N \rightarrow M \vee N$ is of the form $h=$ $f \circ p+\left\langle\left(h_{I J}\right)\right\rangle$ for some $f: M b N \rightarrow M \vee N$.

Using this fact and the dual to Lemma 3, which we do not bother to record, we can prove.

THEOREM 2'. If $h_{\circ} i=\left(h_{I J}\right): M \vee N \rightarrow M \times N$ is an invertible matrix, then $h: M \vee N \rightarrow M \vee N$ is a homotopy equivalence.

Proof. It follows from Lemma $3^{\prime}$ that

$$
h^{\sharp}:[M \vee N, Y] \rightarrow[M \vee N, Y]
$$

is an isomorphism, provided that $Y$ has a multiplication. Since this applies to the Eilenberg-MacLane spaces $Y=K(G, n)$, we see that $h: M \vee N \rightarrow M \vee N$ is a map between simply-connected $C W$ complexes which induces isomorphisms on singular cohomology and hence is a homotopy equivalence.

THEOREM 4'. Let $i_{\sharp}:[M \vee N, M \vee N] \rightarrow[M \vee N, M \times N]$ be a multiplicative homomorphism. Then

(i ) $h: M \vee N \rightarrow M \vee N$ is a homotopy equivalence if and only if $h \circ i=\left(h_{I J}\right): M \vee N \rightarrow M \times N$ is an invertible matrix, and

(ii) there is a short exact sequence of multiplicative groups

$$
1 \rightarrow[M \vee N, M b N] \rightarrow G(M \vee N) \rightarrow G L\left(2, \Lambda_{I J}\right) \rightarrow 1 .
$$

THEOREM 6 . The function $i_{\ddagger}:[M \vee N, M \vee N] \rightarrow[M \vee N, M \times N]$ is a multiplicative homomorphism if and only if

$$
[M \vee N, M b N] \circ p \circ[M \vee N, M \vee N] \circ i=0,
$$

equivalently, kernel $i_{\sharp}=[M \vee N, M b N] \circ p$ is a left ideal in $[M \vee N$, $M \vee N]$.

Corollary $7^{\prime}$. If the co-H-spaces $M$ and $N$ satisfy $[M \vee N$, $M b N]=0$ then the group of self-homotopy equivalences of $M \vee N$ is given by 


$$
G(M \vee N)=G L\left(2, \Lambda_{I J}\right)
$$

ExAMPLE 8. If $M=S^{n}=N(n>1)$, then $[M \vee N, M b N]=$ $\pi_{n+1}\left(S^{n} \times S^{n}, S^{n} \vee S^{n}\right) \oplus \pi_{n+1}\left(S^{n} \times S^{n}, S^{n} \vee S^{n}\right)=0$ and the ring structure of $\Lambda_{I J}=\left[S^{n}, S^{n}\right](I, J=M, N)$ coincides with that of $Z$. So the group $G\left(S^{n} \vee S^{n}\right)$ of self-homotopy equivalences of $S^{n} \vee S^{n}$ is given by $G L(2, Z)$, the group of matrices with integer entries and determinant \pm 1 . This shows that two exact sequences derived in Example 3 coincide with those obtained by P. J. Kahn [1, Proposition 2].

We thank the referee for suggested improvements in various imprecise and obscure passages of an earlier version of this paper.

\section{BIBLIOGRAPHY}

1. P. J. Kahn, Self-equivalences of $(n-1)$-connected $2 n$-manifolds, Bull. Amer. Math. Soc. 72 (1966), 562-566.

Received February 12, 1970, and in revised form March 30, 1970.

The University of Oregon 


\section{PACIFIC JOURNAL OF MATHEMATICS}

\section{EDITORS}

H. SAMELSON

Stanford University

Stanford, California 94305

\section{Richard Pierce}

University of Washington

Seattle, Washington 98105
J. DugundJI

Department of Mathematics

University of Southern California

Los Angeles, California 90007

RICHARD ARENS

University of California

Los Angeles, California 90024

\section{ASSOCIATE EDITORS}

\section{E. F. BeCKenBACH}

B. H. NeUmanN
K. YosHida

\section{SUPPORTING INSTITUTIONS}

\author{
UNIVERSITY OF BRITISH COLUMBIA \\ CALIFORNIA INSTITUTE OF TECHNOLOGY \\ UNIVERSITY OF CALIFORNIA \\ MONTANA STATE UNIVERSITY \\ UNIVERSITY OF NEVADA \\ NEW MEXICO STATE UNIVERSITY \\ OREGON STATE UNIVERSITY \\ UNIVERSITY OF OREGON \\ OSAKA UNIVERSITY \\ UNIVERSITY OF SOUTHERN CALIFORNIA
}

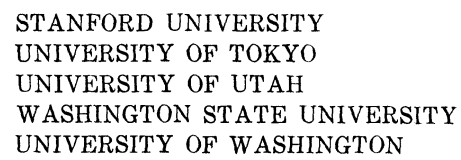

STANFORD UNIVERSITY UNIVERSITY OF TOKYO

UNIVERSITY OF UTAH

WASHINGTON STATE UNIVERSITY

UNIVERSITY OF WASHINGTON

The Supporting Institutions listed above contribute to the cost of publication of this Journal, but they are not owners or publishers and have no responsibility for its content or policies.

Mathematical papers intended for publication in the Pacific Journal of Mathematics should be in typed form or offset-reproduced, (not dittoed), double spaced with large margins. Underline Greek letters in red, German in green, and script in blue. The first paragraph or two must be capable of being used separately as a synopsis of the entire paper. The editorial "we" must not be used in the synopsis, and items of the bibliography should not be cited there unless absolutely necessary, in which case they must be identified by author and Journal, rather than by item number. Manuscripts, in duplicate if possible, may be sent to any one of the four editors. Please classify according to the scheme of Math. Rev. Index to Vol. 39. All other communications to the editors should be addressed to the managing editor, Richard Arens, University of California, Los Angeles, California, 90024.

50 reprints are provided free for each article; additional copies may be obtained at cost in multiples of 50 .

The Pacific Journal of Mathematics is published monthly. Effective with Volume 16 the price per volume (3 numbers) is $\$ 8.00$; single issues, $\$ 3.00$. Special price for current issues to individual faculty members of supporting institutions and to individual members of the American Mathematical Society: $\$ 4.00$ per volume; single issues $\$ 1.50$. Back numbers are available.

Subscriptions, orders for back numbers, and changes of address should be sent to Pacific Journal of Mathematics, 103 Highland Boulevard, Berkeley, California, 94708.

PUBLISHED BY PACIFIC JOURNAL OF MATHEMATICS, A NON-PROFIT CORPORATION

Printed at Kokusai Bunken Insatsusha (International Academic Printing Co., Ltd.), 7-17, Fujimi 2-chome, Chiyoda-ku, Tokyo, Japan. 


\section{Pacific Journal of Mathematics}

\section{Vol. 34, No. 3 \\ July, 1970}

Richard Hindman Bouldin, The peturbation of the singular spectrum

Hugh D. Brunk and Søren Glud Johansen, A generalized Radon-Nikodym derivative .

Henry Werner Davis, F. J. Murray and J. K. Weber, Families of $L_{p}$-spaces

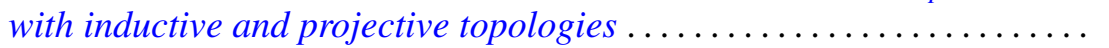

Esmond Ernest Devun, Special semigroups on the two-cell .

Murray Eisenberg and James Howard Hedlund, Expansive automorphisms

of Banach spaces ......................................

Frances F. Gulick, Actions of functions in Banach algebras.

Douglas Harris, Regular-closed spaces and proximities.

Norman Lloyd Johnson, Derivable semi-translation planes . .

Donald E. Knuth, Permutations, matrices, and generalized Young

tableaux..........................................

Herbert Frederick Kreimer, Jr., On the Galois theory of separable

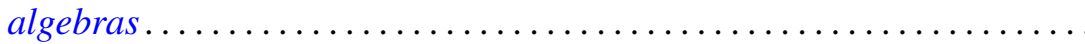

You-Feng Lin and David Alon Rose, Ascoli's theorem for spaces of

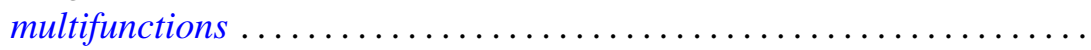

David London, Rearrangement inequalities involving convex functions . . . .

Louis Pigno, A multiplier theorem.

749

Helga Schirmer, Coincidences and fixed points of multifunctions into trees.

755

Richard A. Scoville, Some measure algebras on the integers .

Ralph Edwin Showalter, Local regularity of solutions of Sobolev-Galpern

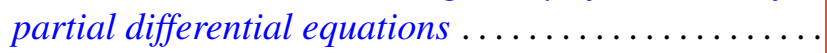

Allan John Sieradski, Twisted self-homotopy equivalences

John H. Smith, On S-units almost generated by S-units of subfields ...

803

Masamichi Takesaki, Algebraic equivalence of locally normal

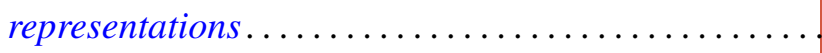

Joseph Earl Valentine, An analogue of Ptolemy's theorem and its converse in

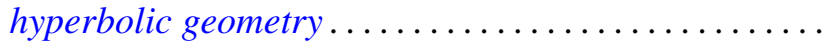

David Lawrence Winter, Solvability of certain p-solvable linear groups of finite order 